\title{
Adoção de restauração com degradação diferenciada em redes EONs para a recuperação de desastres
}

\author{
Gabriel B. Regis ${ }^{1}$, Keiko V. O. Fonseca ${ }^{1}$, Gustavo B. Figueiredo ${ }^{2}$, Juliana de Santi ${ }^{1}$ \\ ${ }^{1}$ DAINF - Universidade Tecnológica Federal do Paraná (UTFPR) - Curitiba \\ ${ }^{2}$ Departamento de Ciência da Computação - Universidade Federal da Bahia (UFBA) \\ gabrielregis@alunos.utfpr.edu.br, keiko@utfpr.edu.br \\ gustavo@dcc.ufba.br, jsanti@utfpr.edu.br
}

\begin{abstract}
This paper investigates the use of restoration for desaster recovery in Elastic Optical Networks (EONs). The proposed algorithms consider different requirements on bandwidth degradation and restoration delay while restoring conections in a gradual and non-gradual way. The main contribution of the proposed approachs is allow a better adaptation of connection recover process to the post-disaster network conditions and consequently, increase the number of restored connections. To assess the performance of the proposed algorithms, comparisons with an approach of literature are performed. Results show improvement on restoration of connections and reduction of blocking rate, which highlights the benefits of the proposed algorithms.
\end{abstract}

Resumo. Neste trabalho, investiga-se a utilização de restauração para a recuperação de desastres em Redes Ópticas Elásticas (Elastic Optical Networks - EONs). Os algoritmos propostos consideram diferentes requisitos de degradação de banda passante e atraso de restauração enquanto restauram conexões de forma gradual e não-gradual. A principal contribuição das abordagens propostas é permitir uma melhor adaptação do processo de recuperação de conexão às condições da rede pós-desastre e, consequentemente, aumentar o número de conexões restauradas. Para avaliar o desempenho dos algoritmos propostos foram realizadas comparações com algoritmo de restauração presente na literatura. Os resultados demonstram melhorias na capacidade de restauração e na redução da probabilidade de bloqueio, o que evidencia os benefícios dos algoritmos propostos.

\section{Introdução}

Tecnologias ópticas, como as Redes Ópticas Elásticas (Elastic Optical Networks - EONs), são soluções atraentes para suportar o rápido crescimento da Internet [Cisco 2017] em relação à expansão do acesso banda larga, à diversidade de aplicações e novos serviços oferecidos, à qualidade de serviço e à eficiência energética. A tecnologia EON aumentou a eficiência espectral através da utilização de comutadores e transceptores com grade de transmissão flexível, o que possibilita a transmissão de conexões com diferentes granularidades por meio da adaptação do espectro óptico aos requisitos de tráfego demandados, gerando, assim, canais com largura de banda variável. 
Apesar da grande disponibilidade de banda passante e capacidade de adaptação, desastres em grande escala (como por exemplo, desastres naturais devido à furacões, terremotos, etc., ou ataques maliciosos) podem afetar a infraestrutura óptica com múltiplas falhas diretas ou indiretas nos enlaces e equipamentos. Estas falhas ocasionam severa escassez de recursos, interrupção massiva de conexões e perda de grande quantidade de dados, independentemente de sua relevância ou prioridade [Habib et al. 2013]. Além disso, o tráfego gerado pela operação de recuperação do desastre pode levar a grande flutuação de tráfego e reduzir ainda mais a disponibilidade de recursos da rede na fase pós-desastre. Desta forma, estratégias para a recuperação de desastres são fundamentais para manter um nível aceitável de disponibilidade/sobrevivência da rede enquanto garantem que tal resiliência é provida com boa relação custo-beneficio considerando a eficiência na utilização dos recursos ópticos.

A recuperação de falhas pode ser provida através de abordagens de proteção [Junior and Drummond 2017], as quais são proativas e baseiam-se na alocação redundante de recursos ópticos, garantindo, assim, 100\% de recuperação. Entretanto, devido à imprevisibilidade de desastres e ao alto custo inerente à proteção, uma vez que os recursos alocados para proteção serão utilizados somente se a falha ocorrer, estas estratégias de recuperação são economicamente impeditivas em cenários de desastres [Bao et al. 2016]. Para reduzir tais custos, operadoras de rede podem usar estratégias reativas baseadas em restauração. Nestas estratégias, nenhum recurso de backup é reservado de forma antecipada, ou seja, conexões interrompidas são re-provisionadas utilizando somente os recursos ópticos disponíveis após o desastre. Consequentemente, as estratégias de restauração são mais eficientes na utilização dos recursos, mas não podem prover $100 \%$ de garantia de recuperação. Dessa forma, o sucesso das estratégias de restauração depende diretamente da eficiência na adaptação dos recursos usados pelas conexões sobreviventes e pelas conexões interrompidas, de forma a recuperar o máximo possível de conexões interrompidas.

A capacidade de adaptação de cada conexão é determinada pelos requisitos da mesma, que por sua vez são especificados em contratos (Service Level Agreements SLAs) com o provedor dos serviços [Agrawal et al. 2017]. Assim, estas especificações são de suma importância para o desenvolvimento de estratégias eficientes de recuperação diferenciada. A literatura apresenta uma série de estudos relacionados ao aprimoramento da recuperação de desastres baseada em restauração. Alguns destes estudos, empregam o conceito de degradação de banda passante das conexões a serem restauradas [Savas et al. 2014, Bao et al. 2016]. Especificamente em [Bao et al. 2016], os autores apresentam um algoritmo para re-provisionamento multi-caminhos com degradação de banda para a recuperação de desastres, onde a restauração das conexões é feita de forma gradual (este algoritmo será descrito na Seção 2). O roteamento multi-caminhos é considerado, também, uma solução com potencial para melhorar o desempenho de sobrevivência de conexões [Rai et al. 2007], pois minimiza as chances de interrupção total de uma conexão que foi distribuída através de diferentes caminhos ópticos. Outra opção para o desenvolvimento de estratégias de recuperação é usar a flexibilidade relacionada ao tempo de re-provisionamento [Ferdousi et al. 2015]. Especificamente para desastres, leva-se em consideração a informação sobre o atraso máximo permitido para a restauração, ou seja, o tempo entre a ocorrência da falha, e consequente interrupção, e o tempo no qual a conexão é re-provisionada. A utilização conjunta de degradação de banda 
passante e atraso na restauração foi proposta em [Lisboa et al. 2017], mas o estudo considera apenas cenário com uma única falha. Por outro lado, a combinação de degradação de banda e atraso na restauração com provisionamento multi-caminhos para recuperação de desastres em Redes Ópticas Elásticas não tem sido considerada.

A presente proposta introduz a restauração para a recuperação de desastres em redes EONS empregando os conceitos de degradação máxima permitida de banda passante e atraso da restauração. Diferentes classes de serviço são consideradas para restaurar cada conexão de acordo com seus requisitos e prioridade especificados. Ao prover restauração diferenciada, pretende-se melhorar a utilização dos recursos remanescentes do desastre e, assim, aumentar a capacidade de restauração de conexões. Para tal, as conexões restauradas podem ter sua banda passante degradada de acordo com as especificações de suas classes. Além disso, conexões interrompidas podem ter a restauração postergada de acordo com suas especificações de tolerância ao atraso na recuperação. Tanto a degradação de banda passante quanto a tolerância ao atraso na recuperação abrem espaço para a restauração de conexões que de outra forma teriam sido perdidas. São propostos dois algoritmos: $i$ ) as conexões interrompidas pelo desastre são restauradas de forma gradual, ou seja, cada conexão interrompida recebe, gradualmente, uma unidade de banda através de múltiplas rotas até atingir a banda mínima necessária para ser restaurada; ii) restauração não-gradual, ou seja, tenta-se prover a banda mínima necessária para a restauração da conexão uma única vez e por uma única rota. Os resultados obtidos mostram que a introdução de restauração diferenciada melhora a capacidade de restauração de conexões, reduz a probabilidade de bloqueio e reduz custo relacionado à conexões bloqueadas e perdidas quando comparada com a abordagem de restauração que não considera diferentes classes de serviço [Bao et al. 2016]. Adicionalmente, constata-se que, embora o algoritmo gradual apresente capacidade de restauração ligeiramente superior ao algoritmo não-gradual para cargas elevadas, sua capacidade de aceitar conexões é menor em decorrência da maior fragmentação de espectro produzida na rede.

Este trabalho é organizado da seguinte forma. Na Seção 2, descreve-se o trabalho que introduz o conceito restauração com degradação gradual sobre múltiplas rotas. A Seção 3 introduz os algoritmos propostos para recuperação de desastres utilizando degradação de banda passante e atraso de restauração combinados com reprovisionamento sobre múltiplas rotas e sobre rota única. A Seção 4 descreve os resultados da avaliação dos algoritmos propostos. Por fim, a Seção 5 apresenta as conclusões.

\section{Restauração gradual de conexões para a recuperação de desastres}

Nesta seção, descreve-se o algoritmo FDM (Fairness-aware Degradation based Multipath re-provisioning), o qual introduz o conceito de restauração gradual de conexões sobre múltiplas rotas para a recuperação de desastres em rede ópticas WDM (Wavelength Division Multiplex) [Bao et al. 2016].

No algoritmo FDM, a banda de cada conexão sobrevivente (ou seja, aquela não atingida pelo desastre) é degradada para um comprimento de onda, e cada conexão interrompida recebe um comprimento de onda. Então, as conexões sobreviventes e as conexões interrompidas pelo desastre recebem de forma gradual e cíclica um comprimento de onda a cada passo do processo de restauração. A ideia da atribuição gradual é proporcionar uma distribuição justa de recursos, dando igual chance de restauração para todas 
as conexões. A ordem em que as conexões são consideradas no processo de restauração é determinada pelo coeficiente de degradação, definido da seguinte forma:

$$
\alpha_{k}=\frac{\text { BandaAtual }_{k}}{\text { BandaRequisitada }_{k}}
$$

onde BandaAtual define a quantidade de banda passante que a conexão $k$ tem atualmente e BandaRequisitad $a_{k}$ determina a quantidade de banda passante requisitada por $k$. Então, as conexões são ordenadas em ordem crescente de $\alpha$. Dessa forma, no processo de atribuição gradual são consideradas primeiro as conexões com maior degradação de banda. O coeficiente de degradação é usado, também, para determinar o momento em que a conexão deixa de ser considerada no processo de restauração gradual, o que ocorre em duas situações: $i$ ) a conexão recebeu toda banda requisita $(\alpha \geq 1)$ e é, consequentemente, restaurada; $i$ i) não há possibilidade de restaurar a conexão pois não há banda disponível para a atribuição em nenhuma de seus rotas candidatas. São consideradas $K=3$ rotas disjuntas, previamente definidas, como candidatas para cada par origem-destino. Portanto, cada conexão pode ser restaurada sobre múltiplas rotas.

\section{Algoritmos propostos}

Os algoritmos propostos nesta seção, denominados Differentiated Restoration based Multipath (DiffRM) e DiffRM-Não-Gradual, capitalizam na flexibilidade provida por conexões de diferentes classes de serviço, as quais permitem diferentes níveis de degradação de banda passante e tolerância ao atraso na restauração. Dessa forma, é possível aumentar o número de conexões restauradas durante o processo de recuperação de desastres. São considerados níveis de degradação de banda e tolerância ao atraso na restauração necessariamente dentro dos limites permitidos pelo contrato de SLA de cada classe de serviço (Seção 3.2).

Os algoritmos utilizam um grafo auxiliar $G$ para representar os recursos ópticos disponíveis na rede após um evento de desastre. De maneira similar a [Bao et al. 2016], para cada par origem-destino são consideradas as $K=3$ menores rotas disjuntas précalculadas. O algoritmo DiffRM tenta restaurar o conjunto de conexões interrompidas atribuindo alternadamente à cada conexão uma unidade de banda passante (ou seja, de forma gradual) através de suas $K$ rotas, ou seja, ele pode utilizar roteamento multicaminhos. Por outro lado, o algoritmo DiffRM-Não-Gradual tenta atribuir toda a banda necessária para restaurar uma determinada conexão, e só após restaurar ou perder esta conexão, a próxima conexão é considerada no processo de restauração. O algoritmo DiffRM-Não-Gradual pode usar qualquer umas das $K$ rotas, porém o roteamento para a restauração de uma determinada conexão é feito sobre uma única rota.

As seções a seguir descrevem o modelo de estabelecimento de solicitações de conexão, as classes de serviço usadas e a descrição dos algoritmos propostos.

\subsection{Estabelecimento das requisições de conexão}

No cenário considerado neste trabalho, as requisições para estabelecimento de conexão são classificados em classes de serviço (Class of Services - CoS) de acordo com sua tolerância à degradação de banda passante e à tolerância ao atraso de restauração (Seção 3.2). 
As requisições para estabelecimento de conexão chegam dinamicamente na rede. Cada requisição $r(b, h, s, d, C, D B, A R)$ especifica a banda passante demandada $(b)$, o tempo de duração da conexão $(h)$, o nó de origem $(s)$, o nó de destino $(d)$, a classe de serviço a qual pertence $(C)$, seu nível de tolerância à degradação de banda $(D B)$ bem como seu nível de tolerância ao atraso de restauração $(A R)$. Neste trabalho, o conceito de classes e prioridades é utilizado somente para a restauração das conexões interrompidas por falhas causadas por desastres e não influenciam na forma como os serviços são provisionados na rede. A política de alocação de slots utilizada é a First-Fit. Toda vez que uma nova solicitação para estabelecimento de conexão chega na rede, uma das $K$ menores rotas com recursos disponíveis é selecionada, ou seja, a conexão é estabelecida usando uma única rota. Caso não seja possível estabelecer a conexão em alguma destas rotas, a requisição para estabelecimento de conexão é bloqueada.

\subsection{Prioridades, requisitos e custos de conexões}

Após um evento de desastre a infraestrutura da rede é seriamente comprometida e, consequentemente, a disponibilidade de recursos ópticos pode não ser suficiente para recuperar todas as conexões atingidas. Neste contexto, o uso de uma política de restauração diferenciada é essencial para melhorar a capacidade de restauração de conexões enquanto satisfaz as especificações de SLA mediante a crise de recursos. Considerando os conceitos de classificação em níveis de serviço usadas em [Develder et al. 2012, Bao et al. 2017, Lisboa et al. 2017, Lourenco et al. 2017], a Tabela 1 ilustra as prioridades, os requisitos e os custos (de bloqueio/perda) de conexões consideradas neste trabalho.

Tabela 1. Prioridades, requisitos e custos de conexões

\begin{tabular}{ccccc}
\hline Tempo real & Prioridade & $\begin{array}{c}\text { Degradação de } \\
\text { banda (DB) }\end{array}$ & $\begin{array}{c}\text { Atraso de } \\
\text { restauração }(\mathrm{AR})\end{array}$ & $\begin{array}{c}\text { Custo de bloqueio ou } \\
\text { perda }(\$ /(\mathrm{Gbit} \times \mathrm{km}))\end{array}$ \\
\hline Hard & Urgente & Não permitida & Não permitido & 0.00000375 \\
Soft-bandwidth & Alta & {$[10 \%-50 \%]$} & Não permitido & 0.000003 \\
Soft-delay & Média & Não permitida & {$[10 \%-50 \%]$} & 0.0000015 \\
Non & Baixa & {$[10 \%-50 \%]$} & {$[10 \%-50 \%]$} & 0.0 \\
\hline
\end{tabular}

A tabela apresenta quatro classes de serviço nomeadas considerando-se o fato de serem ou não de tempo real: Hard, Soft-bandwidth, Soft-delay e Non-real-time. Toda vez que um desastre é detectado, as conexões da classe Hard, que precisam ser recuperadas imediatamente (ou seja, o atraso na restauração não é permitido) com a banda total requisitada (ou seja, degradação de banda não é permitida), são prioritárias no processo de restauração. As conexões da classe Soft-bandwidth são sensíveis ao atraso na restauração, mas permitem a redução de sua banda para um valor entre $10 \%$ e $50 \%$ da banda passante requisitada. Por outro lado, as conexões da classe Soft-delay permitem que a restauração seja postergada por um período de tempo entre $10 \%$ e $50 \%$ da duração residual da conexão (ou seja, o tempo restante para a finalização da transmissão da conexão), mas sua recuperação deve ser realizada considerando a banda total demandada. Por sua vez, as conexões da classe Non-real-time possuem a menor prioridade e, consequentemente, são consideradas somente depois da restauração das conexões de todas as outras classes. Além disso, para as conexões desta classe é possível realizar tanto degradação de banda quanto atraso na restauração, sendo a classe mais flexível e que, portanto, proporciona as melhores oportunidades a serem exploradas por nossos algoritmos. 


\subsection{Algoritmos para restauração de conexões interrompidas}

Os algoritmos propostos são executados sempre que um conjunto $D$ de conexões são interrompidas devido à múltiplas falhas causadas por um evento de desastre. No Algoritmo DiffRM (Algoritmo 1), as conexões são restauradas levando-se em consideração as prioridades e os requisitos descritos na Seção 3.2. A degradação de banda passante ocorre para conexões das classes Soft-bandwidth e Non-real-time. Além disso, para conexões da classe Non-real-time a restauração pode ser postergada somente após uma tentativa mal sucedida de degradação de banda. Por outro lado, as conexão da classe Soft-delay não permitem degradação de banda, mas sua restauração pode ser postergada quando não há recurso suficiente para restaurá-las.

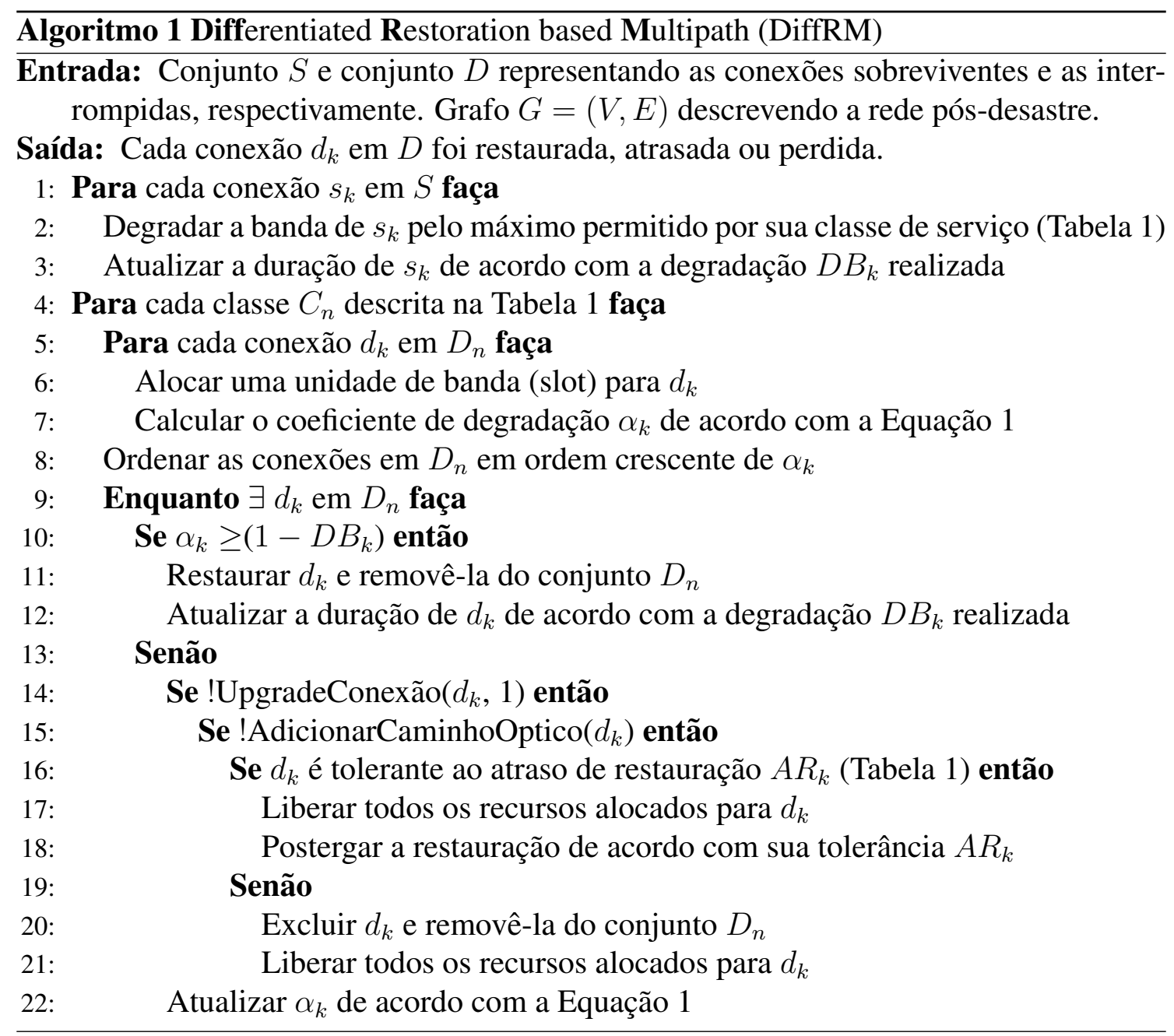

Após um evento de desastre, o primeiro passo para o sucesso do processo de restauração é adaptar a rede de forma a aumentar a disponibilidade de recursos e, assim, abrir espaço para restaurar as conexões interrompidas. Para tal, na Linha 2, as conexões sobreviventes (conjunto $S$ ), ou seja, aquelas que não foram atingidas pelo desastre, são submetidas à degradação de banda passante de acordo com o nível de tolerância especificado em suas respectivas classes de serviço $\left(D B_{k}\right)$. Neste caso, há um aumento no tempo de duração da transmissão da conexão (Linha 3) para compensar a redução na sua taxa de transmissão. Diferentemente do algoritmo FDM (Seção 2), o algoritmo DiffRM degrada 
a banda somente pela quantidade permitida pela classe, garantindo assim que conexões sobreviventes não sejam excluídas por não atingirem a banda passante necessária.

O processo de restauração de conexões interrompidas é realizado para cada classe $C_{n}$ da Tabela 1 (Linha 4) assegurando que as conexões da classe Hard tem precedência sobre as conexões das classes Soft-bandwidth, Soft-delay e Non-real-time. Da mesma forma, as conexões da classe Soft-bandwidth tem precedência sobre as conexões das classes Soft-delay e Non-real-time. Por sua vez, as conexões da Soft-delay tem precedência sobre àquelas da classe Non-real-time. Na Linha 6 , caso haja disponibilidade de recursos, cada conexão interrompida $d_{k}$ em $D_{n}$ (conjunto das conexões interrompidas da classe $C_{n}$ ) recebe um slot de banda passante em uma de suas $K$ rotas alternativas. Adicionalmente, o coeficiente de degradação $\alpha_{k}$ para cada conexão interrompida $d_{k}$ é definido de acordo com a Equação 1 (Linha 7). O coeficiente de degradação é usado para verificar a quantidade de banda passante recebida por cada conexão durante o processo de atribuição gradual de banda (Linha 10). Levando em consideração os valores de $\alpha_{k}$, o conjunto das conexões interrompidas $D_{n}$ é ordenado em ordem crescente (Linha 8) para assegurar que as conexões com maior degradação de banda tenham prioridade nas etapas de atribuição gradual de banda para a restauração (Linhas 9-Linha 22). O processo de atribuição de banda é feito de forma gradual e cíclica para cada conexão $d_{k}$ a ser restaurada. Semelhante à abordagem FDM (Seção 2), a atribuição gradual e cíclica é uma tentativa de prover distribuição justa da banda disponível e, consequentemente, dar chance de restauração para todas as conexões interrompidas da classe $C_{n}$. Se uma conexão $d_{k}$ alcança a banda mínima especificada $\left(1-D B_{k}\right)$ (Linha 10), ela é restaurada e removida do conjunto das conexões interrompidas (Linha 11). Além disso, sua duração é ajustada de acordo com sua taxa de transmissão (Linha 12). Caso a banda mínima especificada ainda não tenha sido alcançada, a banda de $d_{k}$ é incrementada por um slot em alguma das $K$ rotas já usadas por ela (Linha 14) ou em uma nova rota (Linha 15) dentre as $k$ rotas alternativas. Caso exista falta de recursos para atribuir para $d_{k}$, mas há tolerância ao atraso de restauração $A R_{k}$ (Linha 16), a restauração da conexão é postergada e uma nova tentativa de restauração é escalonada de acordo com o valor de tolerância $A R_{k}$ especificado (Linha 18). Ademais, os recursos anteriormente alocados para $d_{k}$ são liberados para as conexões ainda não restauradas (Linha 17). Quando não há recurso disponível nem tolerância ao atraso de restauração, a conexão é considerada perdida (excluída da rede), ela é removida do conjunto $D_{n}$ (Linha 20), e seus recursos de banda passante são liberados (Linha 21). Após cada etapa da atribuição gradual, o valor do coeficiente de degradação $\alpha_{k}$ é atualizado (Linha 22) de forma a detectar se a conexão já alcançou sua banda mínima para ser restaurada na próxima etapa do processo de restauração.

A cada chegada de uma conexão $d_{k}$, que teve sua restauração postergada, se há um caminho com pelo menos $b_{k}$ unidades de banda disponível, ou seja, a banda requisitada, a conexão é restaurada. Caso não exista tal caminho, mas $d_{k}$ é uma conexão da classe Nonreal-time, há ainda a flexibilidade de degradação de banda, e se for possível encontrar um caminho com a banda mínima especificada $\left(1-D B_{k}\right)$, a conexão é restaurada e seu tempo de duração é ajustado para contemplar a degradação de taxa de transmissão. Caso contrário, a restauração se torna impraticável uma vez que não é possível garantir nem mesmo a banda mínima aceitável.

O algoritmo DiffRM-Não-Gradual funciona da mesma forma que o algoritmo Dif- 
fRM entre a Linha 1 e a Linha 4. As demais linhas são substituídas por:

5: $\quad$ Ordenar as conexão interrompidas $\left(D_{n}\right)$ da classe $C_{n}$ em ordem decrescente de banda requisitada $b_{k}$

6: $\quad$ Enquanto $\exists d_{k}$ em $D_{n}$ faça

7: $\quad$ Se $\exists l \mid B(l) \geq\left(b_{k} * D B_{k}\right)$ então

8: $\quad$ Restaurar $d_{k}$, removê-la de $D_{n}$ e atualizar sua duração de acordo com $D B_{k}$

9: $\quad$ Senão

10: $\quad$ Excluir $d_{k}$ e removê-la de $D_{n}$

Assim, no algoritmo DiffRM-Não-Gradual, são restauradas primeiro as conexões com maior banda requisitada (Linha 5) e a restauração é realizada se é possível encontrar toda a banda mínima demandada em um caminho $l$ uma única vez (Linha 7), ou seja, de forma não-gradual e em uma única rota.

\subsubsection{Análise de complexidade}

Algoritmo DiffRM: considerando que a menor taxa de transmissão é $X$ slots, uma capacidade de $Y$ slots por subportadora e $E$ enlaces na rede, podem existir no máximo $(Y / X) \times E$ conexões no conjunto $S$, então há $\mathcal{O}((Y / X) \times E)$ operações na Linha 1 , onde $Y / X$ é uma constante. Da mesma forma, podem existir $(Y / X) \times Z$ conexões no conjunto $D$, onde $Z$ é uma constante representando o número de enlaces atingidos pelo desastre. Assim, na Linha 5 existem $\mathcal{O}((Y / X) \times Z)$ operações. Embora os elementos do conjunto $D$ sejam ordenados (Linha 8 ), seu custo também é constante uma vez que existem no máximo $(Y / X) \times Z$ conexões em $D$. Por sua vez, a Linha 9 é definida pelo número de conexões em $D$ multiplicado pelo número de vezes que cada conexão tem sua banda gradualmente incrementada. Considerando que a taxa máxima de uma conexão é $H$ slots, então cada conexão pode ser incrementada no máximo $(H-1)$ vezes, e na Linha 9 são executadas $\mathcal{O}((Y / X) \times Z) \times H)$ operações, onde $H$ é uma constante. Consequentemente, na Linha 4 existem $\mathcal{O}(((Y / X) \times Z)+((Y / X) \times Z)+(((Y / X) \times Z)) \times H) \times C$ operações, com a constante $C$ representando o número de classes usadas. Assim, a complexidade do algoritmo DiffRM é $\mathcal{O}(E)$.

Algoritmo DiffRM-Não-Gradual: a complexidade do algoritmo DiffRM-NãoGradual é determinada pelo número de operação da Linha $1(\mathcal{O}((Y / X) \times E))$ somado ao número de operação da Linha 4 . O número de operações na Linha 4 é definido pelo número de classes $C$ usadas multiplicado pela soma das operações da Linha 5 e da Linha 6 , as quais são determinadas pelo número de conexões $(Y / X) \times Z \mathrm{em} D$. Assim, a complexidade do algoritmo DiffRM-Não-Residual é $\mathcal{O}((Y / X) \times E)+\mathcal{O}(C \times((Y / X) \times Z))$, que é igual a $\mathcal{O}(E)$ já que todas os demais termos são constantes.

\section{Resultados Numéricos}

Para avaliar o desempenho dos algoritmos propostos foram realizadas simulações e comparações com o algoritmo FDM [Bao et al. 2016] e sua variação não gradual (FDMNão-Gradual), os quais não usam diferentes níveis de serviço para a recuperação de desastres. Para este fim, utilizou-se o simulador de eventos discretos ONS [Costa et al. 2017].

O método de replicação independente foi utilizado para gerar intervalos de confiança com nível de confiança de 95\%. Dez simulações para cada carga entre 50 
Erlangs e 230 Erlangs, com saltos de 20 unidades, foram executadas. Em cada execução, foram consideradas 100000 requisições de conexão distribuídas uniformemente entre todos os pares origem-destino, com as seguintes granularidades: 40Gbps, 50Gbps, 60Gbps, 70Gbps e 80Gbps [Bao et al. 2016]. A duração de cada conexão segue uma distribuição exponencial negativa com média de 60 unidades de tempo [Savas et al. 2014]. A taxa de chegada de requisições segue uma distribuição de Poisson, e estas requisições são distribuídas entre as classes de serviço apresentadas na Tabela $1 \mathrm{com}$ a seguinte distribuição de probabilidade: 8/18 para Non-real-time; 4/18 para Soft-bandwidth; 4/18 para Softdelay; 2/18 para Hard-real-time [Bao et al. 2017]. Ademais, os níveis de degradação de banda e tolerância ao atraso na restauração são aleatoriamente gerados, respectivamente, no intervalo $[10 \%, 50 \%]$ da banda requisitada e $[10 \%, 50 \%]$ do tempo residual da conexão (ou seja, tempo restante para a finalização da transmissão da conexão).

A topologia USNet, com 24 nós e 43 enlaces bidirecionais (Fig. 1) foi utilizada nas simulações. As quatro áreas demarcadas por círculos pontilhados representam as zonas de desastres consideradas para as simulações [Bao et al. 2016]. Cada zona está atrelada a um instante de tempo, os quais são distribuídos uniformemente na linha do tempo utilizada para cada simulação. Além disso, todos os enlaces e nós atingidos pelo desastre são considerados indisponíveis durante o desastre, e o tempo de restauração é proporcional ao número de nós e enlaces atingidos.

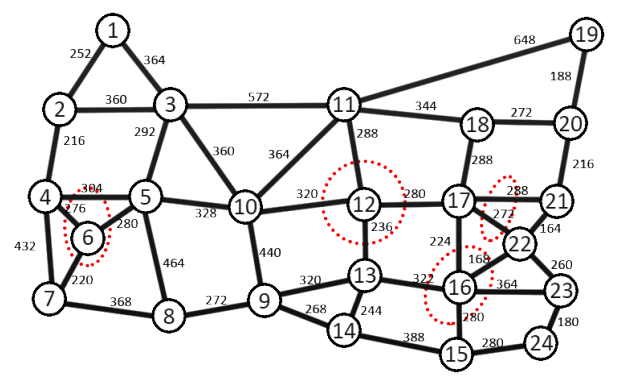

Figura 1. Topologia USNet (baseada em [Bao et al. 2016]).

Uma Rede Óptica Elástica (EON) é simulada, onde o espectro em cada enlace consiste de 156 slots de subportadoras, cada um com $12,5 \mathrm{GHz}$ de frequência. Os formatos de modulação BPSK, QPSK, 8QAM, 16QAM, 32QAM, 64QAM, 128QAM, e 256QAM são utilizados com base na distância da rota utilizada para servir a conexão. A política de alocação de espectro utilizada foi a First-Fit.

As métricas utilizadas para avaliar os algoritmos propostos são: o número médio de conexões não restauradas (ou seja, perdidas); o percentual de conexões não restauradas para cada classe de serviço $(C o S)$; a probabilidade de bloqueio de conexões (BP - Blocking Probability), ou seja, a razão entre o número de solicitações de conexão bloqueadas e o número total de solicitações de conexão; a probabilidade de bloqueio de banda (BBR - Bandwidth Blocking Ratio), ou seja, a razão entre a quantidade de banda bloqueada e o total de banda requisitada; a fragmentação do espectro na rede e o custo financeiro das solicitações de conexão bloqueadas e das conexões perdidas (ou seja, interrompidas não restauradas).

A Figura 2 apresenta o número médio de conexões não restauradas (perdidas) em função da carga na rede. Para cargas de 50 Erlangs, onde 36 conexões foram interrom- 
pidas por eventos de desastre, os algoritmos FDM e FDM-Não-Gradual não conseguiram restaurar 30\% destas conexões, enquanto os algoritmos DiffRM e DiffRM-Não-Gradual não restauraram $25 \%$ e $21 \%$ das conexões interrompidas, respectivamente. Para cargas de 230 Erlangs, as abordagem FDM e FDM-Não-Gradual restauraram, respectivamente, $64 \%$ e $61 \%$ das conexões interrompidas. Por outro lado, os algoritmos DiffRM e DiffRMNão-Gradual obtiveram sucesso na restauração de $75 \%$ e $70 \%$ das conexões interrompidas, respectivamente. A superioridade na capacidade de restauração dos algoritmos propostos deve-se à flexibilidade provida pela restauração diferenciada de conexões. A degradação de banda permitida pelas classes Soft-bandwidth e Non-real-time previnem a perda de conexões pertencentes a estas classes em um momento de escassez de recursos, enquanto mantém um nível mínimo aceitável de qualidade de serviço. Além disso, mediante a indisponibilidade de recursos, a possibilidade de postergar a restauração de conexões, provida pelas classes Soft-Delay e Non-real-time, permite que estas conexões tenham a chance de ser re-provisionadas em um futuro próximo usando banda passante liberada pela finalização da transmissão de outras conexões ou pela reparação da infraestrutura da rede. A ligeira inferioridade na capacidade de restauração apresentada pelos algoritmos Não-Graduais deve-se ao fato de que estas estratégias tentam encontrar a banda necessária para a restauração em uma única interação, o que reduz a chances de sucesso na restauração das demais conexões interrompidas.

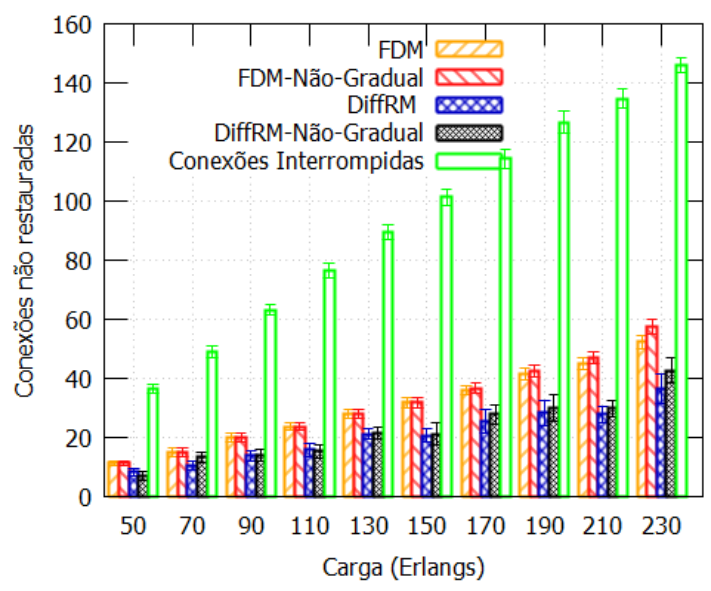

Figura 2. Número de conexões não restauradas em função da carga na rede.

Para aprofundar a investigação sobre o impacto da restauração baseada em classes com diferentes níveis de serviço, foi realizada uma avaliação da percentagem de conexões não restauradas (DP - Dropping Probability) por classe de serviço $(C o S)$ em função da carga na rede. Os resultados desta avaliação são apresentados na Figura 3 e na Figura 4 para os algoritmos graduais e não graduais, respectivamente. De forma geral, é possível verificar que as abordagens que levam consideração diferentes níveis de serviço para a realização da restauração (DiffRM e DiffRM-Não-Gradual) apresentaram menores taxas de DP comparadas às abordagens que não empregam restauração diferenciada (FDM e FDM-Não-Gradual), o que é justificado pela flexibilidade provida pela degradação de banda e atraso de restauração. Além disso, para os algoritmos com degradação diferenciada (DiffRM e DiffRM-Não-Gradual) são produzidos valores de DP diferentes para cada classe de serviço, enquanto que para os algoritmo FDM e FDM-Não-Gradual, independentemente das características e requisitos (Hard, Soft-bandwidth, Soft-delay e Non), 
as conexões são excluídas praticamente na mesma proporção. Na Figura 3, para cargas

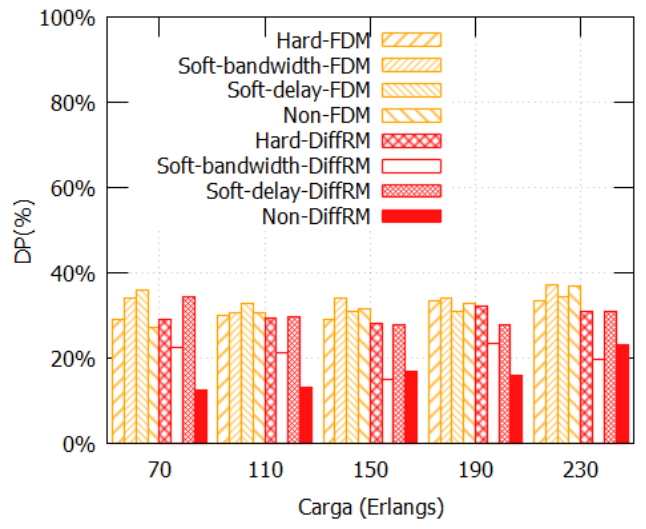

Figura 3. Percentagem de conexões não restauradas (perdidas) por $C o S$ em função da carga na rede - abordagens graduais.

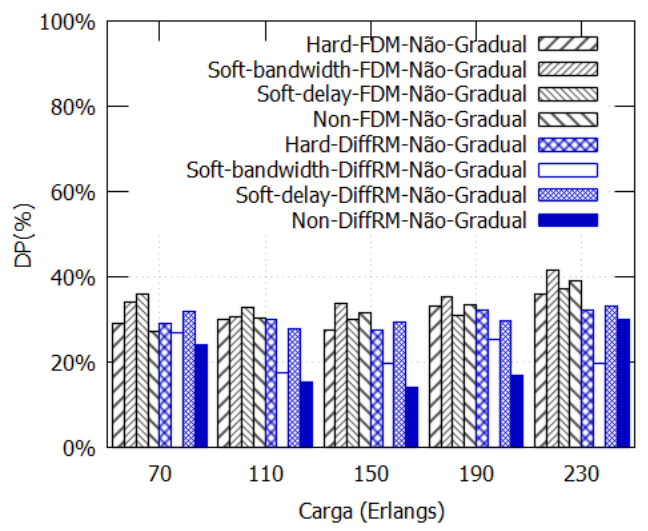

Figura 4. Percentagem de conexões não restauradas (perdidas) por $C o S$ em função da carga na rede - abordagens não-graduais.

de 230 Erlangs e conexões da classe Soft-bandwidth, o algoritmo FDM apresentou uma taxa de DP de $37.7 \%$ contra $21 \%$ gerada pelo algoritmo DiffRM. É necessário lembrar que, diferentemente do algoritmo FDM, ao tratar de conexões da classe Soft-bandwidth, o algoritmo DiffRM considera uma conexão restaurada assim que a banda mínima especificada (Tabela 1) é atribuída à conexão. Ao analisar os valores gerados para a classe Soft-delay, que tolera o atraso de restauração, o algoritmo FDM produziu uma taxa de DP de $34.4 \%$ em comparação à $31 \%$ gerado pelo algoritmo DiffRM. Isso ocorre pois o segundo algoritmo implementa a tolerância ao atraso de restauração, permitindo que uma nova tentativa de restauração possa ser realizada futuramente, o que é de grande relevância em cenários de indisponibilidade de recursos espectrais. É importante notar ainda que as conexões da classe Non-real-time apresentam a maior redução nos valores de DP, evidenciando a importância de se explorar a flexibilidade provida pelas especificações de SLA de cada classe.

A Figura 5 e a Figura 6 apresentam, respectivamente, a probabilidade de bloqueio de solicitações de conexão (BP) e a probabilidade de bloqueio de banda (BBR), em função da carga na rede. Em função dos baixos valores de carga, os quais foram usado de forma a gerar um cenário fiel ao do algoritmo FDM usado na comparação, e da flexibilidade da tecnologia EON, foram gerados pequenos valores de BP e BBR. No entanto, é importante notar o comportamento das linhas que representam os algoritmos DiffRM-Não-Gradual e FDM-Não-Gradual. Nossa hipótese para este comportamento é que ao restaurar as conexões atribuindo espectro de forma gradual, as algoritmos DiffRM e FDM acabam por produzir maior fragmentação de recursos [Horota et al. 2014], ou seja, são gerados pequenos fragmentos não-contíguos no espectro, que dessa maneira, não podem ser usados para acomodar novas solicitações de conexão. Considerando-se a Equação 2 [Horota et al. 2014], os valores para a fragmentação da rede gerados pelos algoritmos avaliados neste trabalho são apresentados na Figura 7. Pode-se observar que para todas as cargas consideradas, os valores de fragmentação gerados pelos algoritmos DiffRM-Não-Gradual e FDM-Não-Gradual são menores do que àqueles produzidos por 


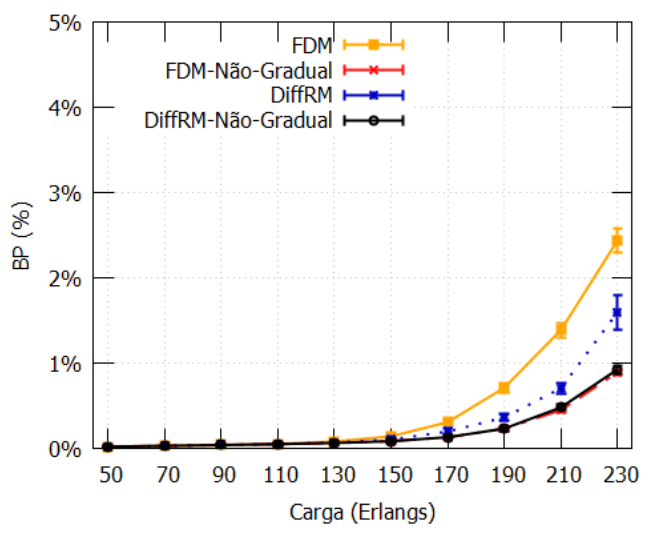

Figura 5. BP em função da carga na rede.

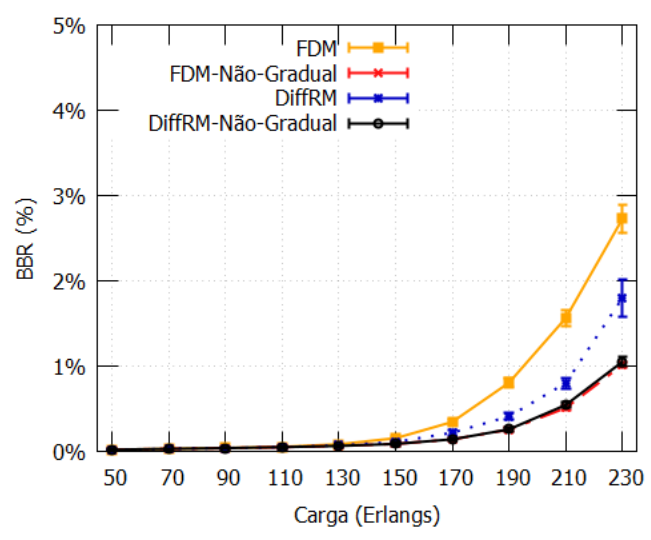

Figura 6. BBR em função da carga na rede.

suas versões de restauração gradual. Para a carga de 230 Erlangs, os algoritmos DiffRMNão-Gradual e FDM-Não-Gradual geram, respectivamente, fragmentação 85.5\% e 94.4\% menores do que suas versão graduais. A investigação sobre o impacto da fragmentação em cenários de desastres será aprofundada pelo autores em trabalho futuro.

$$
F=\frac{\text { maior BlocoLivre }}{\text { totalLivre }}
$$

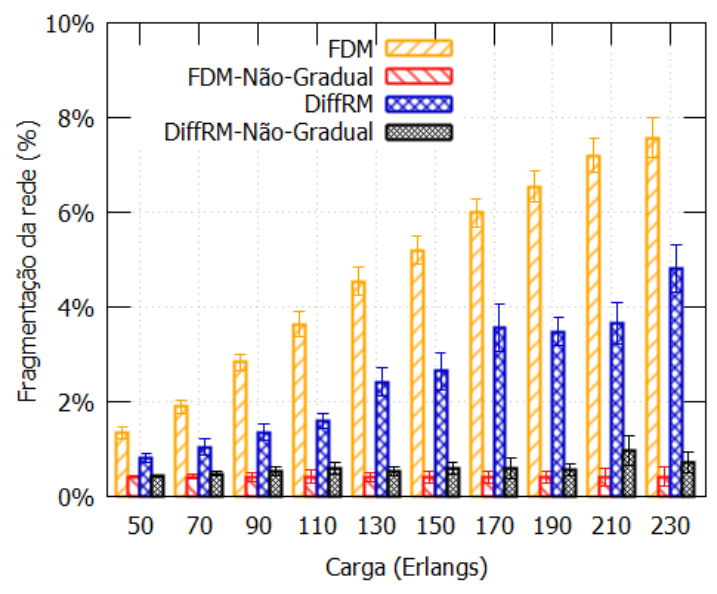

Figura 7. Fragmentação da rede em função da carga na rede.

Considerando os valores da quinta coluna na Tabela 1 [Lourenco et al. 2017], o impacto financeiro, em função da carga na rede, gerado pelo bloqueio de solicitações de conexão e pela não restauração (perda) de conexões interrompidas por desastres são apresentados na Figura 8 e na Figura 9, respectivamente. Ao falharem na aceitação de novas solicitações de conexão, os algoritmos propostos geraram um custo até 70\% (DiffRMNão-Gradual) e até 34\% (DiffRM) menores do que o algoritmo FDM para cargas de 230 Erlangs. Como o algoritmos FDM-Não-Gradual e DiffRM-Não-Gradual apresentam baixo valores de probabilidade de bloqueio (Figura 5), seus custos são equivalentes ao bloqueio gerado. Os custos em relação à falha na restauração das conexões interrompidas 
(Figura 9) apresentados pelos algoritmos propostos são aproximadamente $30 \%$ menores do que os custos gerados pelo algoritmo FDM para cargas de 230 Erlangs. Estes valores mostram a importância, principalmente do ponto de vista das operadoras de rede, da tomada de decisões para reduzir custos de bloqueio/perda de conexões e, consequentemente, maximizar lucros.

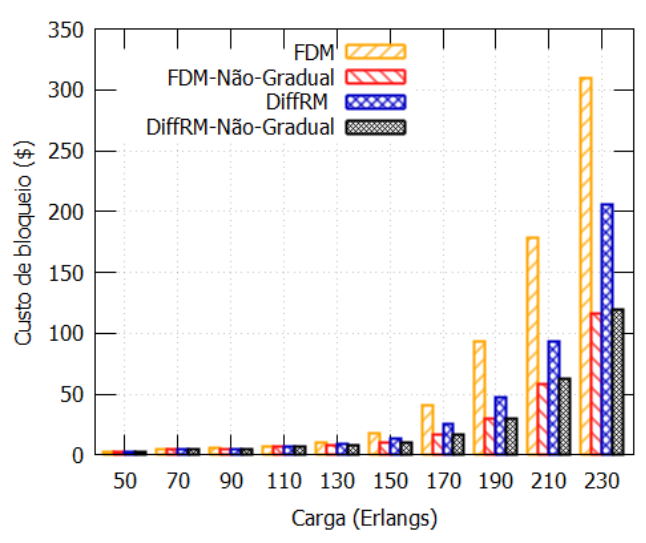

Figura 8. Custo do bloqueio de requisições em função da carga na rede.

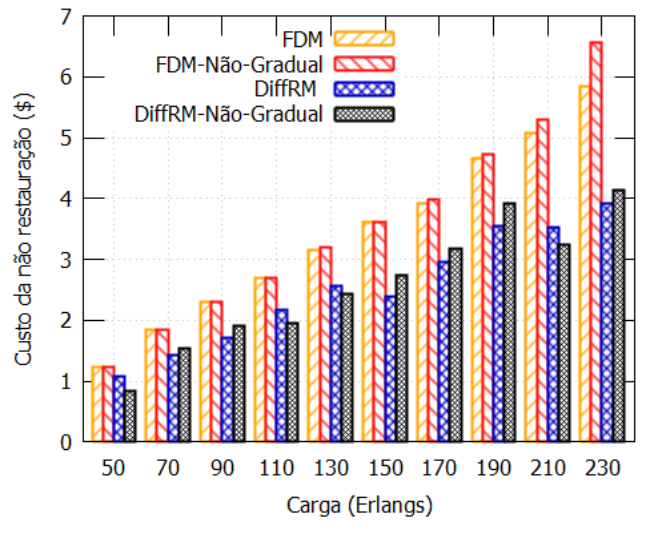

Figura 9. Custo da não restauração em função da carga na rede.

\section{Conclusão}

A grande disponibilidade de banda provida pelas redes ópticas impulsionou a diversidade de serviços e aplicações, que por vez demandam diferentes níveis de qualidade de serviço. Estes níveis são explicitamente especificados em contratos SLA, os quais possibilitam maior conhecimento de informações a respeito das conexões, tais como degradação de banda passante e tolerância ao atraso de restauração. Estas informações podem ser utilizadas para gerenciar e controlar os recursos ópticos no momento de recuperação de desastres.

Neste trabalho, apresentou-se dois algoritmos de restauração para a recuperação de desastres em redes ópticas EONs. São duas as principais contribuições deste trabalho: i) a utilização de classes com diferentes níveis de degradação de banda passante e tolerância ao atraso de restauração como base para prover restauração diferenciada e, assim, aproveitar ao máximo os recursos disponíveis em cenários com escassez de recursos; ii) a investigação sobre o impacto da restauração gradual sobre a capacidade de restaurar conexões interrompidas e sobre o bloqueio de novas requisições. Resultados de simulações indicam que os algoritmos propostos são superiores em capacidade de restauração, redução de bloqueio de conexões, redução do bloqueio de banda e redução do custo monetário quando comparados ao algoritmo com restauração gradual sem diferenciação de classes. Além disso, verificou-se que embora a abordagem não-gradual aumente ligeiramente o número de conexões não restauradas para valores elevados de carga, ela reduz a probabilidade de bloqueio de banda e a probabilidade de bloqueio de conexões.

\section{Agradecimentos}

Suporte: CNPq-Universal (processos 402805/2016-0 e 420907/2016-5), Secure Cloud (processo 2549). Apoio: H2020-ICT-2015 EU-BR, RNP, MCTI e NVIDIA Corporation. 


\section{Referências}

Agrawal, A., Vyas, U., Bhatia, V., and Prakash, S. (2017). SLA-aware differentiated QoS in elastic optical networks. Optical Fiber Technology, 36(Supplement C):41 - 50.

Bao, N. H., Tornatore, M., Martel, C. U., and Mukherjee, B. (2016). Fairness-aware degradation based multipath re-provisioning strategy for post-disaster telecom mesh networks. IEEE/OSA Journal of Optical Comm. and Networking, 8(6):441-450.

Bao, N.-H., Wu, Y.-K., Su, G.-Q., Yuan, Y., and Luo, D.-Y. (2017). Hierarchical fairness based re-provisioning in post-disaster telecom networks. International Conference on Computer, Information and Telecommunication Systems, pages 330 - 333.

Cisco (2017). The Zettabyte Era - Trends and Analysis. https://www.cisco.com/c/en/us/solutions/collateral/service-provider/visualnetworking-index-vni/vni-hyperconnectivity-wp.html. Último acesso: 19-03-2018.

Costa, L. R., da Silva, K. A., de Sousa, L. S., and Drummond, A. C. (2017). ONS - optical network simulator WDM/EON. http://comnet.unb.br/en/grupos/get/ons. 21-01-2018.

Develder, C., Leenheer, M. D., Dhoedt, B., Pickavet, M., Colle, D., Turck, F. D., and Demeester, P. (2012). Optical networks for grid and cloud computing applications. Proceedings of the IEEE, 100(5):1149-1167.

Ferdousi, S., Tornatore, M., Habib, M. F., and Mukherjee, B. (2015). Rapid data evacuation for large-scale disasters in optical cloud networks [invited]. IEEE/OSA Journal of Optical Communications and Networking, 7(12):B163-B172.

Habib, M. F., Tornatore, M., Dikbiyik, F., and Mukherjee, B. (2013). Disaster survivability in optical communication networks. Comp. Communications, 36(6):630 - 644.

Horota, A. K., Figueiredo, G. B., and Fonseca, N. L. S. (2014). Algoritmo de Roteamento e Atribuição de Espectro com Minimização de Fragmentação em Redes Ópticas Elásticas. Simp. Brasileiro de Redes de Comp. e Sist. Distribuídos (SBRC), pages 1-14.

Junior, P. J. S. and Drummond, A. C. (2017). Proteção por Pré-provisionamento em Redes Ópticas Elásticas. Workshop em Desempenho de Sistemas Computacionais e de Comunicação (SBC - WPerformance), pages 1-14.

Lisboa, F., Fonseca, K. V. O., Vieira, L. C., Monti, P., Figueiredo, G. B., and de Santi, J. (2017). Restoration Based on Bandwidth Degradation and Service Restoration Delay for Optical Cloud Networks. In IEEE Global Communications Conference (GLOBECOM), pages 1-6.

Lourenco, R. B. R., Tornatore, M., Martel, C. U., and Mukherjee, B. (2017). Running the Network Harder: Connection Provisioning with Degradation under Resource Crunch. ArXiv e-prints.

Rai, S., Deshpande, O., Ou, C., Martel, C. U., and Mukherjee, B. (2007). Reliable multipath provisioning for high-capacity backbone mesh networks. IEEE/ACM Transactions on Networking, 15(4):803-812.

Savas, S. S., Habib, M. F., Tornatore, M., Dikbiyik, F., and Mukherjee, B. (2014). Network adaptability to disaster disruptions by exploiting degraded-service tolerance. IEEE Communications Magazine, 52(12):58-65. 